\title{
Design and Development of Web Based Embedded Robot for
}

\section{Safety and Security Applications}

\author{
${ }^{1}$ Dr. Subodh Kumar Panda, ${ }^{2}$ M Harshita, ${ }^{3}$ Nishmitha A R, ${ }^{4}$ Priyanka P \\ ${ }^{1}$ Associate Professor, Department of Electronics and Communication Engineering, B N M Institute of \\ Technology, Bengaluru, Karnataka, India
}

${ }^{2}$ UG Student, Department of Electronics and Communication Engineering, B N M Institute of Technology,

Bengaluru, Karnataka, India

${ }^{3}$ UG Student, Department of Electronics and Communication Engineering, B N M Institute of Technology,

Bengaluru, Karnataka, India

${ }^{4}$ UG Student, Department of Electronics and Communication Engineering, B N M Institute of Technology,

Bengaluru, Karnataka, India

${ }^{1}$ subodhpanda2013@gmail.com, ${ }^{2}$ harshitam2703@gmail.com, ${ }^{3}$ nishmithaar21@ @mail.com, ${ }^{4}$ priyanka143 1999@gmail.com.

\begin{abstract}
This paper proposes the design and development of an embedded system with a robot for safety and security application using wifi communication and web server. This compact design has robot along with various peripherals and sensors for detecting hazardous gas leakage and performs necessary control actions like alarm for fire accidents, intruder detection and sends alert message to the local system. The robot can extinguish fire and monitors the area inside industry and passes the audio-video streaming to the local system. This robot also has a battery powered wireless AV camera which provides robotic in front environment information to the local and remote system and performs the audio and video streaming through web server.
\end{abstract}

\section{INTRODUCTION}

In recent years, robots have become more popular and customary in various sectors. Robots have the capability to perform the mission, without any damage, greater chances of success in dangerous environment, sustainability, reliability, etc. An embedded system is meant to perform specific control functions within a bigger system with temporal constraints. The robot is simple to design and implement both in hardware and software aspects. Robots are no longer confined to industrial automation only and also used in many other applications. Robots are becoming increasingly reliable, affordable and user friendly. Robots are designed for performing everyday responsibilities like vacuum cleaning, child monitoring and private assistance etc. thereby improving the quality of life. Medical equipment utilizing controller microchips that translate muscle movements into prosthetic responses. As the demand for these robots grows, so does the requirement for qualified professionals.

In a typical mechanical oriented task, robots are equipped with sensors, actuators, and software to perceive their environment and safely perform programmed goals. An embedded system resides inside the robot tying together the various subsystems. Without an embedded system, robots would want to depend upon external computing systems which might increase the protection risks thanks to delay and failure within the communication link between the robot and its external system.

\section{RELATED WORK}

[1] The research work presented by Mustafa and Dilsad describes a line following robot which is an autonomous robot which is able to follow either a black line that is drawn on the surface consisting of a 
contrasting color. It is designed to move automatically and follow the line. The robot uses arrays of optical sensors to identify the line, thus assisting the robot to stay on the track. The array of four sensor makes its movement precise and flexible.

[2] V. Ramya and B. Palaniappan discussed in detail about how the toxic gases have to be monitored; such that increase in the normal level of them could be known and proper precaution measures can be taken. An embedded system is designed using PIC 16F877 microcontroller, for the purpose of detection of hazardous gas leakage. The system also supports to provide real-time monitoring of concentration of the gases which presents in the air.

[3] This paper discusses about an autonomous agent for gas leak source detection. The robot estimates the localization of the gas leak source in an indoor environment without any human intervention. The agent implements an SLAM (simultaneous localization and mapping) procedure to scan and map the indoor area. The robot samples gas concentrations with a gas sensor in order to estimate the source of the gas leak. The robot will use the info from the on board sensors in order to define an efficient scanning path.

[4] The author discussed about building a system which can be used universally at any scale to monitor the parameters in the environment. Raspberry-pi and sensors collects all the real-time data from environment and this real-time data is fetched by the web server and display it. User can access this data from anywhere through Internet. Wireless sensor networks (WSN) has been employed to collect data about physical phenomenon in various applications such as habitat monitoring.

[5] Mohd Aliff and Samsiah Sani discussed in detail about how to detect and extinguish the fire sensed. Development of a firefighting robot that can extinguish fire without the need for fire fighters to be exposed to unnecessary danger. It is designed to be compact in size than other conventional fire-fighting robot in order to ease small location entry for deeper reach of extinguishing fire in narrow space.

In all the above work the researches have worked on different applications of robot like line following, detection of hazardous gas leakage and firefighting robots. But there is no single design available where the robot can be used for multiple purposes so there is a necessity to develop a robotic embedded system for multiple purposes like monitoring the gas leakage, detect intruder and extinguish fire in night when no one is available in the industry and send the critical information to the local system using wifi module.

\section{METHODOLOGY}

In this work an embedded system robot is designed for safety and security purpose using wifi communication. It is an autonomous line-controlled robot that detects the leakage of hazardous gases such as LPG, methane, natural gas etc. in industries and raises alarm. The robot also performs necessary control actions like alarm for fire accidents, intruder detection and sends alert message to the local system. The robot can extinguish fire and monitors the area inside industry and passes the audio-video streaming to the local system. A low-cost microcontroller and other peripherals such as a high sensitivity gas sensors, IR photo transistors, ultrasonic (PING) sensors, PIR sensors, wireless AV camera and wifi module is used to serve multiple purposes. The microcontroller is embedded with $\mathrm{C}$ program that processes the sensors data and provides safety and secure information through wifi communication. The whole system is compact and robot is battery powered. Figure 1 shows the block diagram of web based embedded robot for safety and security system.

Blynk software platform is used to quickly build interfaces for controlling and monitoring hardware from iOS and android devices. By using Blynk application, it is possible to create a project dashboard and arrange buttons, sliders, graphs, and other widgets onto the screen. An IP camera software is used for audio and video streaming at the local station.

The login page at the local system can be accessed only by the authenticated user by entering the username and password. At local system, the information received from robot can be viewed in the dashboard and the robotic movement can be controlled. 


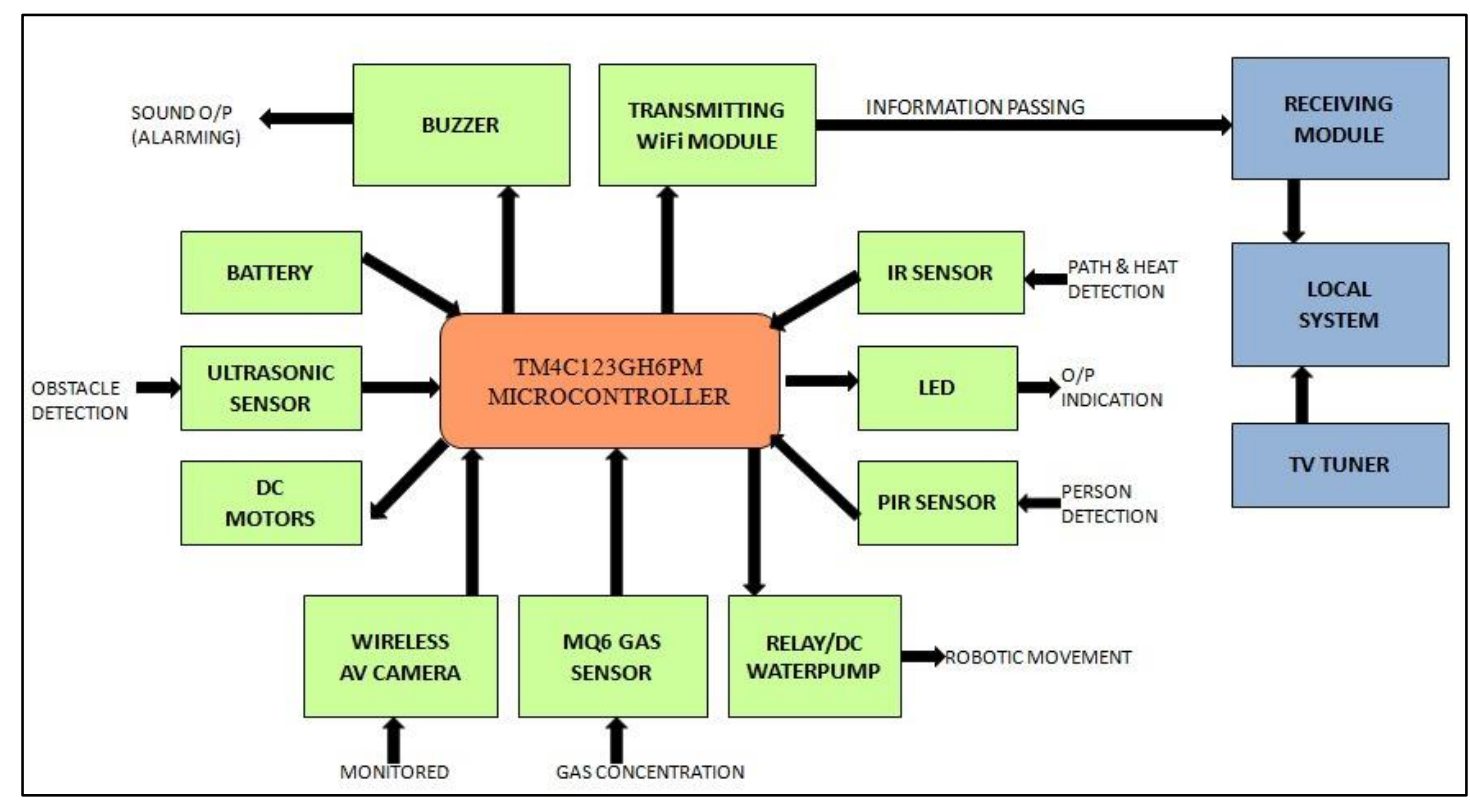

Figure 1: Block diagram of web based embedded robot for safety and security system

The MQ6 sensor is used for detection of hazardous gases like LPG, iso-butane, propane and LNG in the range of 200 to $10000 \mathrm{ppm}$. The gas detection is indicated by LED present on-board.

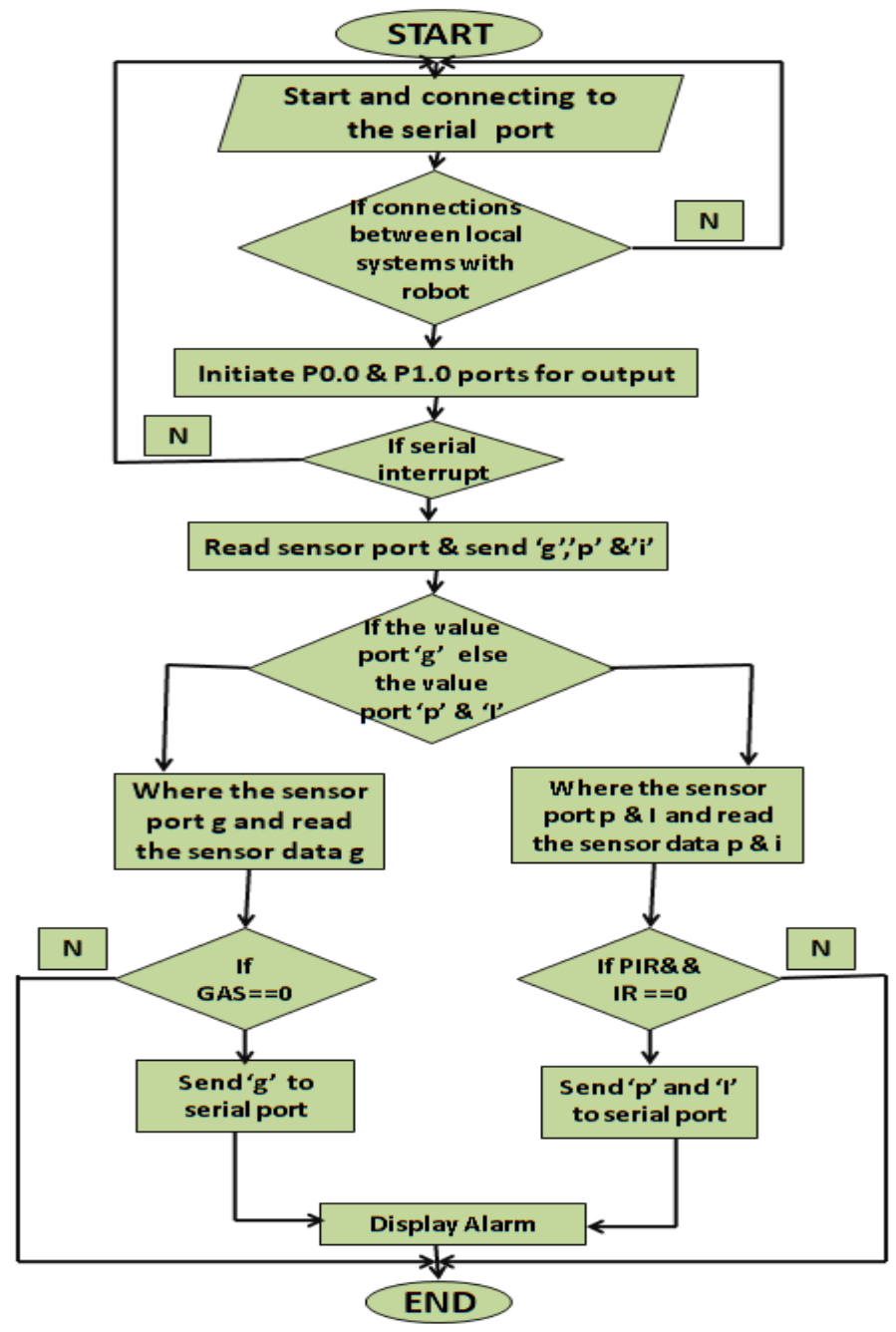

Figure 2: Flowchart for Gas, PIR and IR sensor module 
Simultaneously a buzzer is beeped to alarm the people working around and the robot is moved to the place where the gas leakage occurred. Also an alert message "Gas leakage detected" is sent to the local system. This alert information is highly helpful to avoid the disaster due to life threatening hazardous gas leakage. The IR photo-transistor present in robot detects the fire and an on board extinguisher is used to stop the fire automatically and raise an alarm, also sends an alert message as "Fire detected" to the local system. Thus, ensuring the lives of people working and living around the area. The intruder detection uses a PIR sensor. PIR sensor senses the presence of human being by infrared radiations. If someone present it sends alert message as "'Motion detected" to the local system. The video and audio streaming is done to ensure no person is entering to the hazardous area and also to know the location of robot. The low-cost wireless AV camera at the robot captures its in-front audio and video information and performs AV streaming at the local system.

The Node-MCU is operated on battery power with sleep mode which reduces the battery use. There is less probability of interfering with other users, since it incorporates CSMA-CA protocol and also supports automatic re transmission of data which ensures the robustness of the network. It facilitates ease-of-use and supports larger networks that comprised of thousands of devices. The robot has four wheels which are used for moving backward, forward, left and right which is shown in figure 3.

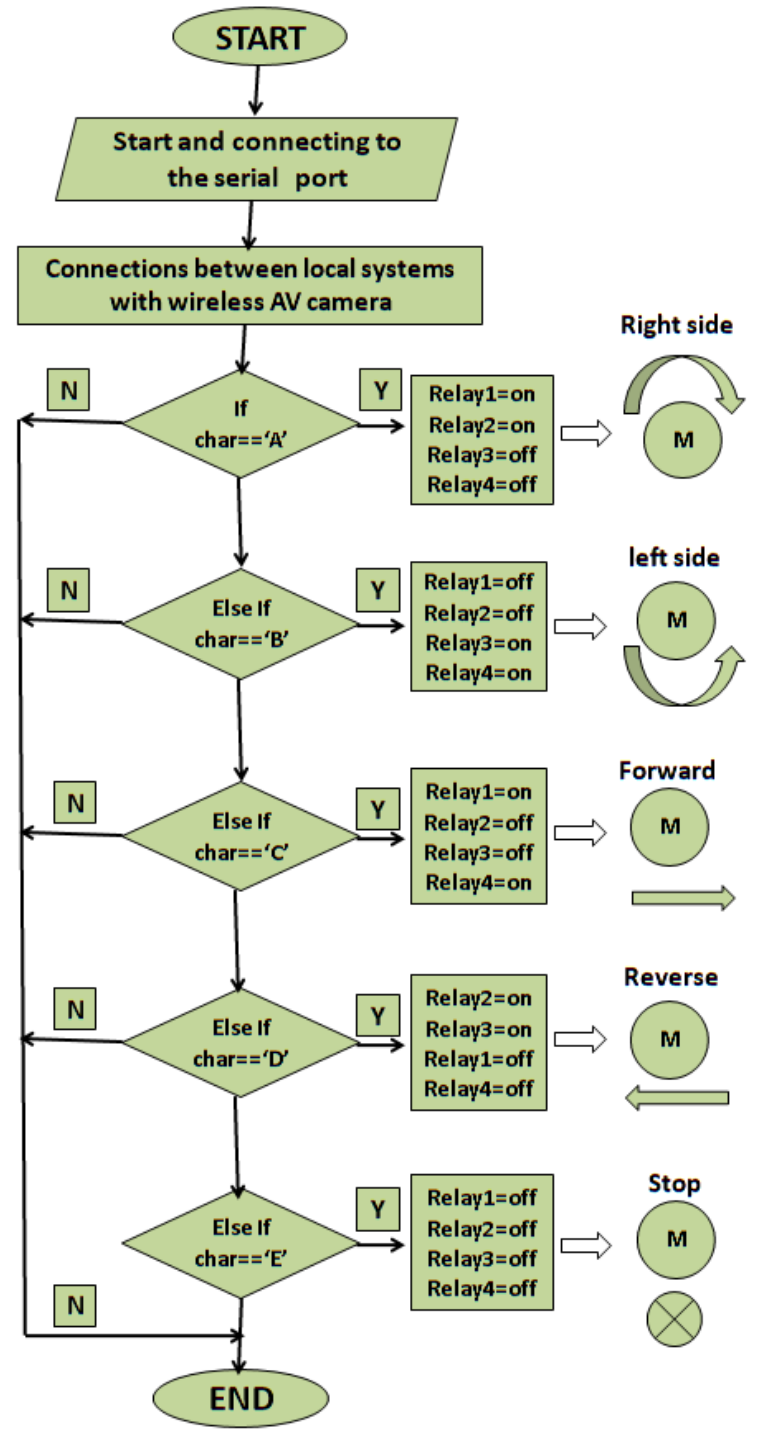

Figure 3: Flowchart for robotic direction control 


\section{RESULTS AND DISCUSSION}

The implementation and result of the proposed system for different cases of the device operation at the local system is discussed below:

CASE 1: Initially when Gas, fire and intruder are not detected

The Blynk app dashboard displays the messages "GAS LOW", "FIRE not Detected" and Motion not Detected" respectively as shown in figure 4.

CASE 2: Only when Gas is detected

The Blynk app dashboard displays the messages "GAS HIGH", "FIRE not Detected" and "Motion not Detected" respectively as shown in figure 5 .

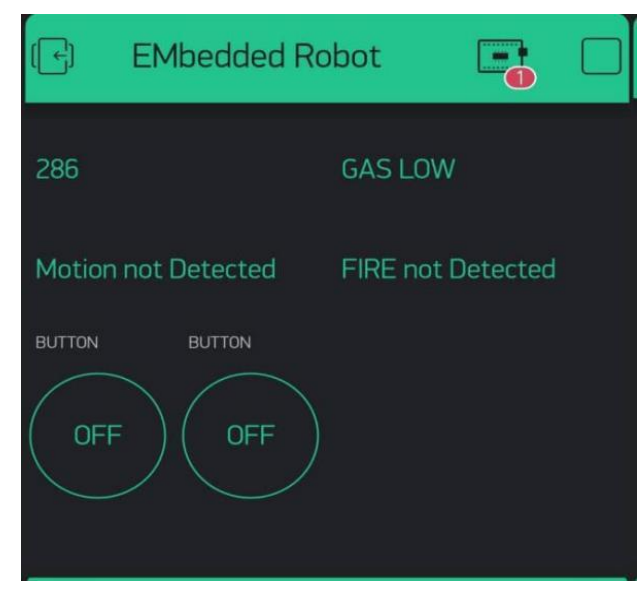

Figure 4: Blynk output for initial conditions when gas, intruder, and fire are not detected.

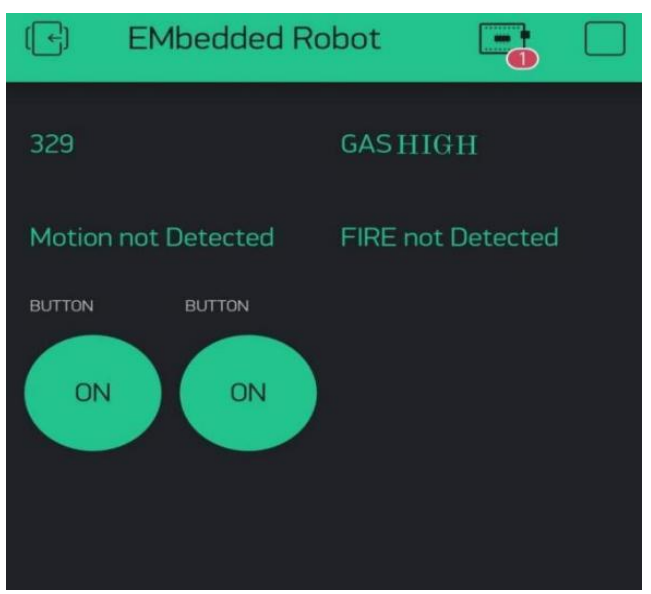

Figure 5: Blynk output when gas concentration is high with no fire and intruder detection.

CASE 3: Only when Intruder detected

The Blynk app dashboard displays the messages "GAS LOW", "FIRE not Detected" and "Motion Detected" respectively as shown in figure 6.

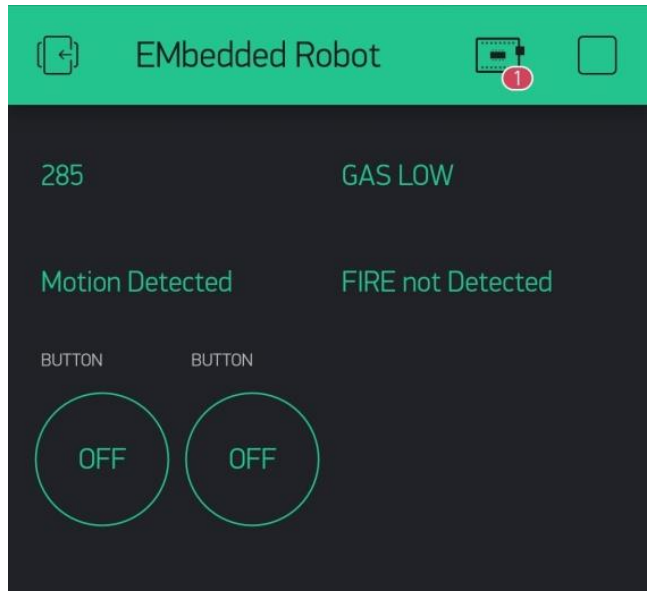

Figure 6: Blynk output when only Intruder is detected.

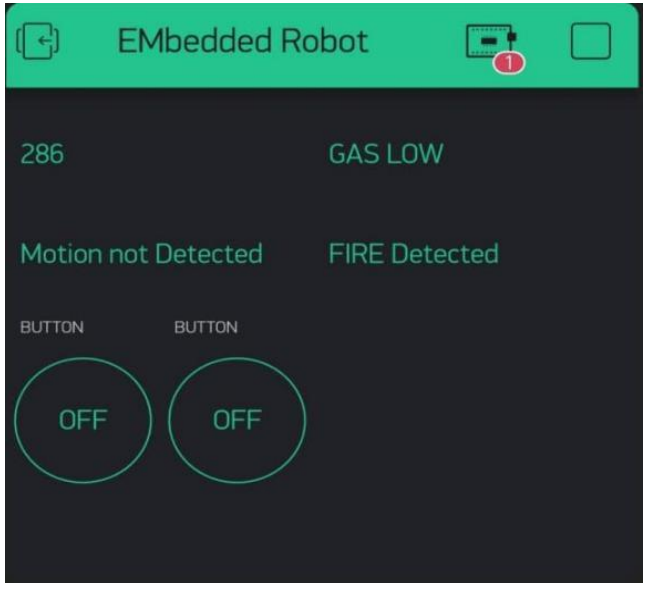

Figure 7: Blynk output when only fire is detected.

CASE 4: Only when fire is detected 
CASE 4: Only when fire is detected

The Blynk app dashboard displays the messages "GAS LOW", "FIRE Detected" and "Motion not Detected" respectively as shown in figure 7.

CASE 5: When both motion and fire is detected

When the gas concentration is low in the surroundings and an intruder is detected during a fire accident the Blynk app dashboard displays the messages "GAS LOW", "FIRE Detected" and "Motion Detected" respectively as shown in figure 8 .

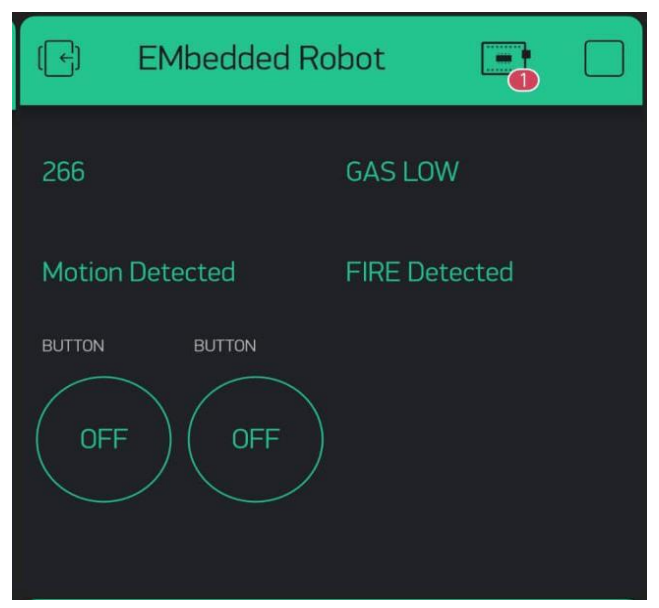

Figure 8: Blynk output when both fire and intruder is detected.

There are two buttons present in the Blynk app which controls the motion of the robot from local system. The working model of web based embedded robot for safety and security system is shown in figure 9.

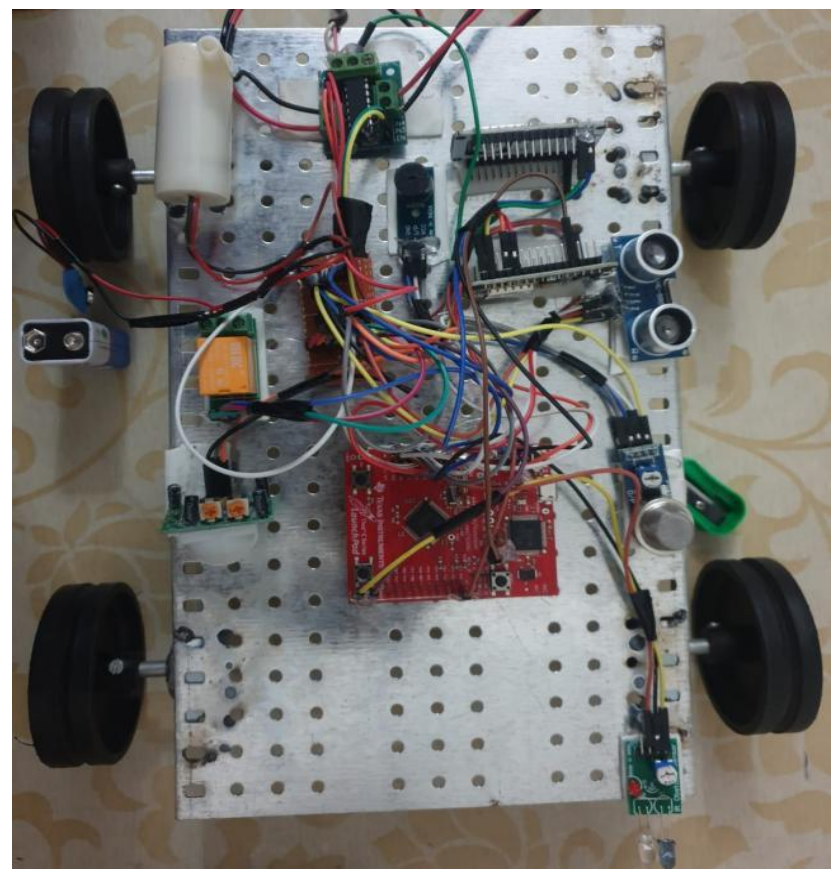

Figure 9: Working model of web based embedded robot for safety and security system

\section{CONCLUSION}

In this work an embedded robot is designed and developed for safety and security application. It is observed that it can detect various hazardous gases and sends alert message to local system. Result shows that the proposed project has significant advantages over the traditional methods. It is observed that the robot performs necessary control actions like alarm for fire accidents, intruder detection and sends alert 
message to the local system. It is observed that, a buzzer is beeped to alarm the people working around and the robot is moved to the place where the gas leakage occurred. The IR photo-transistor present in robot detects the fire and an on board extinguisher is used to stop the fire automatically. A wireless AV camera resides at the robot, sends the robotic environment information to the local system. The video streaming is simultaneously done at both the local and ' $\mathrm{N}$ ' number of remote system (web server). The videos are streamed using the IP camera software, this software is used to create a web page to do live streaming through the web server.

This system can be used where ever the safety and security are the major threat. In future this work may be enhanced in such a way that, whenever a picture is captured then an IP Camera can immediately send an email about the picture. And also, Wifi module may be used to increase the communication distance between the Robot and with the local system.

\section{REFERENCES}

[1] Mustafa Engin1, Dilşad Engin2. "Path planning of line follower robot"

[2] V. Ramya, B. Palaniappan, "Embedded system for Hazardous Gas detection and Alerting", International Journal of Distributed and Parallel Systems (IJDPS) Vol.3, No.3, May 2012.

[3] Dani Martinez, Tomas Palleja, Javier Moreno, Marcel Tresanchez, MerceTeixido, "A Mobile Robot Agent for Gas Leak Source Detection" - Institute for BioEngineering of Catalonia,Baldiri Reixac, 10-16, 08028 Barcelona, Spain

[4] Aarti Rao Jaladi, Karishma Khithani, Pankaja Pawar, Kiran Malvi. "Environmental Monitoring Using Wireless Sensor Networks (WSN) based on IOT"- International Research Journal of Engineering and Technology (IRJET) Volume: 04 Issue: 01

[5] K Medilla \& Firdaus, Firdaus \& Yulianto, Andik \& K Syakban, 2018, Early detection of LPG gas leakage based on Wireless Sensor Networking, https://www.researchgate.net/publication/323451555_Early_detection_of_LPG_gas_leakage_based_W ireless_Sensor_Networking/ accessed on August 26, 2019.

[6] Premkumar M, "Unmanned Multi-Functional robot using zigbee adopter network for defense application” (IJARCET), volume2, Issue I, January 2013.

[7] Shradha Khandekar, Sagar Mergal, Gita Lonkar, Prof A S Zadhuke, "Review on web cam robot using zigbee", (ISSN), E\&TC Dept of SBPOCE, Indapur, volume 3, January 2016.

[8] Hsian-I Lin, Yu-Cheng Liu, "Evaluation of Human-Robot Arm Movement Imitation”, Nat. Taipei university of Technol., Taipei, Taiwan, IEEE, May 2016.

[9] V. Ramya, B. Palaniappan, K. Karthik and Subash Prasad "Embedded System for vehicle cabin toxic gas detection and alerting", Journal of Elsevier Procedia Engineering, 30(2013).

[10] Heng -Tze Cheng, Zheng, Pei Zhang, "Real-Time Imitative Robotic Arm Control for Home Robot Applications", Carnegie Mellon University, IEEE, March 2015.

[11] Cherubini, "Development of a multi-mode navigation system for an assistive robotics project", IEEE International Conference on Robotics and Automation, Rome, Italy, 10-14 April 2014.

[12] Edward B. Panganiban "Automated Hazardous Gas Detecting Robot using Wireless Sensor Networks with GSM-SMS Alert and Fire Control System for Households"Article in International Journal of Advanced Trends in Computer Science and Engineering. June 2019. https://doi.org/10.30534/ijatcse/2019/72832019. 